\title{
How Relevant is Obstetrician and Gynecologist Gender to Women in Saudi Arabia?
}

\author{
Ahmed Hussein Subki (D) \\ Abdullah Khaled Agabawi ${ }^{2}$ \\ Muhab Mohammed Hindi (iD ${ }^{2}$ \\ Nadeem Shafique Butt $\mathbb{D}^{3}$ \\ Mohammed Saad Alsallum (iD) ${ }^{4}$ \\ Rawan Ali Alghamdi ${ }^{2}$ \\ Siham Hussein Subki ${ }^{2}$ \\ Fahad Alsallum (iD) ${ }^{2}$ \\ Abdulaziz Ahmed Alharbi ${ }^{5}$ \\ Yousif Ibrahim Lodhi ${ }^{6}$ \\ Sultan Alandijani' \\ Faten Al-Zaben ${ }^{7}$ \\ Harold G Koenig ${ }^{7,8}$ \\ Ayman M Oraif ${ }^{2}$
}

'Department of Internal Medicine, King Faisal Specialist Hospital and Research Center, Jeddah, Saudi Arabia;

${ }^{2}$ Department of Obstetrics and Gynecology, King Abdulaziz University, Jeddah, Saudi Arabia; ${ }^{3}$ Department of Biostatistics, King Abdulaziz University, Jeddah, Saudi Arabia; ${ }^{4}$ Department of Neurology, King Abdulaziz Medical City, Jeddah, Saudi Arabia; ${ }^{5}$ Department of Emergency Medicine, King Fahad General Hospital, Jeddah, Saudi Arabia; ${ }^{6}$ Department of Internal Medicine, East Jeddah Hospital, Jeddah, Saudi Arabia;

${ }^{7}$ Department of Psychiatry, King Abdulaziz University, Jeddah, Saudi Arabia; ${ }^{8}$ Department of Psychiatry \& Behavioral Sciences, Duke University Medical Center, Durham, North Carolina, USA

Correspondence: Ayman M Oraif Department of Obstetrics and Gynecology, King Abdulaziz University, Jeddah, Saudi Arabia

Tel +966538062000

Email ayman_oraif@yahoo.com
Background: The patient-doctor relationship is one of the most important factors in determining the outcome of healthcare. The first step in establishing this relationship is choosing a physician. This study sought to identify patient preferences concerning the gender of their obstetrics and gynecology (OB-GYN) physician and the effect of religion and society on these choices.

Methods: A cross-sectional study was conducted at the OB-GYN outpatient clinics at King Abdulaziz University Hospital in Jeddah between February 2017 and June 2017. A total of 227 female patients were recruited. Eligible were women ages 18 years or older who had attended the clinic at least three times. A 30-item questionnaire was administered.

Results: Significantly, more female doctors were preferred for pelvic examination in lower income group $(\mathrm{p}=0.003)$, while male doctors were preferred for surgery $(\mathrm{p}=0.010)$ in higher income group. Significantly more male doctors were preferred for pelvic examination and gynecological surgery in $>35$-year age group $(\mathrm{p}=0.015$ and $\mathrm{p}=0.017$, respectively). With regard to predictors, embarrassment was the most significant factor reported for not choosing a male obstetrician/gynecologist (OBGYN) in the younger age group. Nearly three-quarters $(71.2 \%)$ of respondents with age $\leq 35$ reported embarrassment as a factor for not choosing a male OB-GYN; 79.7\% of this subgroup indicated that female doctors were more knowledgeable about women's health issues.

Conclusion: Participants expressed a strong preference for female providers overall, although some women preferred male providers during certain circumstances (gynecologic surgery). Despite these gender preferences, more important to women in their choice of OBGYN provider was the doctor's experience, qualifications, and reputation. Such trends are consistent with those culturally similar countries and in line with trends worldwide. These findings have the potential to significantly impact the personal health for women in Saudi Arabia and elsewhere in the Middle East where religious and cultural traditions are so important in decision-making.

Keywords: gender, preference, obstetrics, gynecologist, patient care

\section{Introduction}

The patient-doctor relationship is one of the most important factors in determining the outcome of healthcare in nearly all specialties. ${ }^{1}$ This is especially true for the relationship between a woman and her obstetrics and gynecology (OB-GYN) physician. Women confide with their physician about many healthcare's issues such as reproductive safety, pregnancy, cancer monitoring, to name a few. As a result, it is of utmost importance that a woman feels comfortable and empowered with their choice of OB-GYN doctor.

The number of female OB-GYN physicians has increased over the years, so women have more choices regarding their healthcare provider. Determinants of 
such choices are often the clinical experience of the provider, cost of care, location of the service, age, and in particular, the gender of provider. ${ }^{2}$

The gender of the provider may play a pivotal role in the likelihood of women seeking cancer screening services, with more patient utilization of the Papanicolaou smear test or requests for the pelvic exam when they have a female provider. ${ }^{3,4}$ In one study in Saudi Arabia, medical student gender was the main factor that influenced patient agreement to student involvement during physical examinations. ${ }^{5}$ There may also be some common attitudes of women toward the gender of their OB-GYN doctor. One study found that female OB-GYNs were viewed as easier to talk to and more knowledgeable and capable of understanding women's health issues than were male OBGYNs. $^{6}$

In general, there is a link between religion and choice of gender of healthcare providers in Islamic Middle Eastern countries, where women typically prefer female physicians $(90 \%)$ compared to males. ${ }^{7}$ The cultural and social life in Saudi Arabia is strictly conservative, where the law requires that women cover themselves using an abaya (a black cloak covering the whole body) and unmarried Saudi women do not live alone. In this country, unmarried women live with their parents or another close family member in the absence of the parents; for cultural and religious reasons, it is unacceptable for unmarried Saudi women to live alone in most cases. Also, it is religiously unacceptable for a woman to be alone with men with whom they have no close blood relationship.

The points made above may present barriers to the patient-doctor relationship when the OB-GYN physician is a male, especially when doing examinations such as a pelvic or breast exam. The present study seeks to obtain a better understanding of women's OB-GYN physician gender preferences and identify the effect of religion on this choice in modern Saudi Arabia. The objective is to improve the healthcare for women in terms of regular gynecologic screening and pregnancy care that may be influenced by the doctor-patient relationship.

\section{Methods}

\section{Design, Setting, and Participants}

We conducted a cross-sectional study of consecutive female patients seen in the outpatient and inpatient settings of Obstetrics and Gynecology services at King Abdulaziz University Hospital in Jeddah, Saudi Arabia, between
February and June 2017. The response rate was greater than $95 \%$ among women who were approached. A total of 227 female patients were recruited. To be eligible, women had to be 18 years of age or older and must have attended the clinic at least 3 times.

\section{Measurements}

A 30-item questionnaire was administered in which women were asked to choose the preferred gender of their OB-GYN in various situations. In addition, perceptions of trust and experience of the physician were examined (Table 1).

\section{Ethical Considerations}

The study was approved by the Institutional Review Board (IRB) of King Abdulaziz University Hospital and the Research Ethics Committee of King Abdulaziz University. The ethics approval number was No. 47-17. Written informed consent was obtained from all participants. This study was conducted in accordance with the Declaration of Helsinki.

\section{Statistical Methods}

Categorical variables were expressed as frequency and percentages. Chi-squared tests of Independence were conducted to explore the association of gender preferences for OB-GYN care with various demographic factors. Multivariate analysis Chi-Squared Automatic Interaction Detection (CHAID) classification algorithm was used to

Table I Demographic Characteristics of the Study Sample

\begin{tabular}{|l|l|c|c|}
\hline \multicolumn{2}{|l|}{} & $\mathbf{n}$ & $\mathbf{( \% )}$ \\
\hline Nationality & Non-Saudi & 62 & $(27.3)$ \\
& Saudi & 165 & $(72.7)$ \\
\hline Age in Years & 35 and less & 146 & $(64.3)$ \\
& 36 and above & 81 & $(35.7)$ \\
\hline Marital Status & Not Married & 16 & $(7.0)$ \\
& Married & 211 & $(93.0)$ \\
\hline No. of Children & Less than 5 & 193 & $(85.0)$ \\
& 5 and above & 34 & $(15.0)$ \\
\hline Education Level & High school or less & 92 & $(40.5)$ \\
& College education & 135 & $(59.5)$ \\
\hline Employed & No & 170 & $(74.9)$ \\
& Yes & 57 & $(25.1)$ \\
\hline Family Income & $\leq 10,000$ SAR & 173 & $(76.2)$ \\
& $>10,000$ SAR & 54 & $(23.8)$ \\
\hline
\end{tabular}


create a decision tree to select the most relevant characteristics related to gender preferences based on demographic information.

\section{Results}

The majority of the participants were of Saudi nationality $(n=165,73 \%)$ and were married $(n=211,93 \%)$. Nearly two-thirds $(66 \%)$ were age 35 or younger. Most participants had fewer than 5 children. Education level was high school or less in $40 \%$. Most were not employed outside the home $(\mathrm{n}=170,76 \%)$ and annual income was less than or equal to 10,000 Saudi Arabian riyals (SAR) in the majority $(n=173,76 \%)$. Further details on sample characteristics are provided in Table 1.

Table 2 presents the responses given by women on factors they considered when choosing a physician for OB-GYN care. Most valued was the "knowledge and experience" of the physician, followed by the physician's reputation. The third most important factor was that the physician was board-certified. Table 3 describes the distribution of gender preferences for OB-GYN care stratified by income level of respondents. Gender preference differed for pelvic exams by income groups, such that female physicians were more likely to be preferred for pelvic exams in women with an income of less than or equal to
10,000 SAR ( $\mathrm{p}=0.003)$. In contrast, male physicians were more likely preferred for gynecological surgery by women with incomes of greater than 10,000 SAR $(p=0.01)$. Embarrassment was the most common reason for not choosing a male gynecologist in both income groups but was more common in the lower-income group ( $\mathrm{p}=0.043)$. Slightly more than one-quarter (26\%) of women preferred a male, non-Saudi OB-GYN in the higher-income group compared to about half that (12\%) in the lower-income group $(\mathrm{p}=0.02)$. No other factors were significant.

Table 4 describes the distribution of gender preference when selecting OB-GYN care by the age of the respondent. Gender preferences for pelvic exam varied significantly by age groups, with more male physicians preferred in the over age 35 group ( $\mathrm{p}=0.015)$. A similar age pattern of preference was observed for gynecologic surgery $(\mathrm{p}=0.017)$. Embarrassment as the reason for not choosing a male OB-GYN was more likely in the younger age group $(\mathrm{p}=0.009$ for gynecology, $\mathrm{p}=0.004$ for obstetrics). No significant doctor's gender preferences were observed by marital status, nationality, education, or parity of the respondents.

Multivariate classification tree analysis was applied to test the significance of various demographic characteristics concerning gender preferences. Only age was significantly

Table 2 Preferences* for the Choice of Doctor for OB-GYN Care by Demographic Characteristics

\begin{tabular}{|c|c|c|c|c|c|c|c|c|}
\hline & & $\begin{array}{l}\text { Knowledge and } \\
\text { Experience }\end{array}$ & Religion & $\begin{array}{c}\text { Board } \\
\text { Certification }\end{array}$ & $\begin{array}{l}\text { Age of } \\
\text { Doctor }\end{array}$ & $\begin{array}{l}\text { Gender of } \\
\text { Doctor }\end{array}$ & Personality & Reputation \\
\hline Nationality & $\begin{array}{l}\text { Non-Saudi } \\
\text { Saudi }\end{array}$ & $\begin{array}{l}90.3 \% \\
93.3 \%\end{array}$ & $\begin{array}{l}32.3 \% \\
22.0 \%\end{array}$ & $\begin{array}{l}38.7 \% \\
50.6 \%\end{array}$ & $\begin{array}{l}12.9 \% \\
14.0 \%\end{array}$ & $\begin{array}{l}19.4 \% \\
18.3 \%\end{array}$ & $\begin{array}{l}25.8 \% \\
23.2 \%\end{array}$ & $\begin{array}{l}43.5 \% \\
51.8 \%\end{array}$ \\
\hline $\begin{array}{l}\text { Age in } \\
\text { Years }\end{array}$ & $\begin{array}{l}35 \text { and less } \\
36 \text { and above }\end{array}$ & $\begin{array}{l}93.2 \% \\
91.3 \%\end{array}$ & $\begin{array}{l}21.9 \% \\
30.0 \%\end{array}$ & $\begin{array}{l}42.5 \% \\
56.3 \%\end{array}$ & $\begin{array}{l}15.1 \% \\
11.3 \%\end{array}$ & $\begin{array}{l}20.5 \% \\
15.0 \%\end{array}$ & $\begin{array}{l}20.5 \% \\
30.0 \%\end{array}$ & $\begin{array}{l}47.3 \% \\
53.8 \%\end{array}$ \\
\hline $\begin{array}{l}\text { Marital } \\
\text { Status }\end{array}$ & $\begin{array}{l}\text { Not Married } \\
\text { Married }\end{array}$ & $\begin{array}{l}87.5 \% \\
92.9 \%\end{array}$ & $\begin{array}{l}18.8 \% \\
25.2 \%\end{array}$ & $\begin{array}{l}50.0 \% \\
47.1 \%\end{array}$ & $\begin{array}{l}6.3 \% \\
14.3 \%\end{array}$ & $\begin{array}{c}6.3 \% \\
19.5 \%\end{array}$ & $\begin{array}{l}18.8 \% \\
24.3 \%\end{array}$ & $\begin{array}{l}68.8 \% \\
48.1 \%\end{array}$ \\
\hline $\begin{array}{l}\text { No. of } \\
\text { Children }\end{array}$ & $\begin{array}{l}\text { Less than } 5 \\
5 \text { and above }\end{array}$ & $\begin{array}{l}93.2 \% \\
88.2 \%\end{array}$ & $\begin{array}{l}22.4 \% \\
38.2 \%\end{array}$ & $\begin{array}{l}45.3 \% \\
58.8 \%\end{array}$ & $\begin{array}{l}13.0 \% \\
17.6 \%\end{array}$ & $\begin{array}{l}18.2 \% \\
20.6 \%\end{array}$ & $\begin{array}{l}22.4 \% \\
32.4 \%\end{array}$ & $\begin{array}{l}46.9 \% \\
64.7 \%\end{array}$ \\
\hline $\begin{array}{l}\text { Education } \\
\text { Level }\end{array}$ & $\begin{array}{l}\text { High school } \\
\text { or less } \\
\text { College } \\
\text { education }\end{array}$ & $\begin{array}{l}88.0 \% \\
95.5 \%\end{array}$ & $\begin{array}{l}33.7 \% \\
18.7 \%\end{array}$ & $\begin{array}{l}47.8 \% \\
47.0 \%\end{array}$ & $\begin{array}{l}12.0 \% \\
14.9 \%\end{array}$ & $\begin{array}{l}19.6 \% \\
17.9 \%\end{array}$ & $\begin{array}{l}21.7 \% \\
25.4 \%\end{array}$ & $\begin{array}{l}52.2 \% \\
47.8 \%\end{array}$ \\
\hline Employed & $\begin{array}{l}\text { No } \\
\text { Yes }\end{array}$ & $\begin{array}{l}91.1 \% \\
96.5 \%\end{array}$ & $\begin{array}{c}30.2 \% \\
8.8 \%\end{array}$ & $\begin{array}{l}46.2 \% \\
50.9 \%\end{array}$ & $\begin{array}{l}15.4 \% \\
8.8 \%\end{array}$ & $\begin{array}{l}20.7 \% \\
12.3 \%\end{array}$ & $\begin{array}{l}23.1 \% \\
26.3 \%\end{array}$ & $\begin{array}{l}47.9 \% \\
54.4 \%\end{array}$ \\
\hline $\begin{array}{l}\text { Family } \\
\text { Income }\end{array}$ & $\begin{array}{l}\leq 10,000 \text { SAR } \\
>10,000 \text { SAR }\end{array}$ & $\begin{array}{l}92.4 \% \\
92.6 \%\end{array}$ & $\begin{array}{l}27.3 \% \\
16.7 \%\end{array}$ & $\begin{array}{l}44.2 \% \\
57.4 \%\end{array}$ & $\begin{array}{c}16.3 \% \\
5.6 \%\end{array}$ & $\begin{array}{l}18.6 \% \\
18.5 \%\end{array}$ & $\begin{array}{l}23.8 \% \\
24.1 \%\end{array}$ & $\begin{array}{l}48.3 \% \\
53.7 \%\end{array}$ \\
\hline
\end{tabular}

Note: *Multiple Response factors. 
Table 3 Comparison of Gender Preferences of Doctor by Income Level

\begin{tabular}{|c|c|c|c|c|c|c|c|c|}
\hline & & \multicolumn{6}{|c|}{ Family Income } & \multirow[b]{3}{*}{ P-value } \\
\hline & & \multicolumn{3}{|c|}{$\leq 10,000$ SAR } & \multicolumn{3}{|c|}{$>10,000$ SAR } & \\
\hline & & $\begin{array}{c}\text { No } \\
\text { Preference }\end{array}$ & Male & Female & $\begin{array}{c}\text { No } \\
\text { Preference }\end{array}$ & Male & Female & \\
\hline Pelvic Exam & $\begin{array}{l}\mathrm{n} \\
(\%)\end{array}$ & $\begin{array}{c}47 \\
(27.2)\end{array}$ & $\begin{array}{c}9 \\
(5.2)\end{array}$ & $\begin{array}{c}117 \\
(67.6)\end{array}$ & $\begin{array}{c}13 \\
(24.1)\end{array}$ & $\begin{array}{c}\text { II } \\
(20.4)\end{array}$ & $\begin{array}{c}30 \\
(55.6)\end{array}$ & 0.003 \\
\hline OB Care & $\begin{array}{l}n \\
(\%)\end{array}$ & $\begin{array}{c}47 \\
(27.2)\end{array}$ & $\begin{array}{c}18 \\
(10.4)\end{array}$ & $\begin{array}{c}108 \\
(62.4)\end{array}$ & $\begin{array}{c}13 \\
(24.1)\end{array}$ & $\begin{array}{c}10 \\
(18.5)\end{array}$ & $\begin{array}{c}31 \\
(57.4)\end{array}$ & 0.284 \\
\hline Gyn. Surgery & $\begin{array}{l}\mathrm{n} \\
(\%)\end{array}$ & $\begin{array}{c}61 \\
(35.3)\end{array}$ & $\begin{array}{c}20 \\
(I I .6)\end{array}$ & $\begin{array}{c}92 \\
(53.2)\end{array}$ & $\begin{array}{c}12 \\
(22.2)\end{array}$ & $\begin{array}{c}15 \\
(27.8)\end{array}$ & $\begin{array}{c}27 \\
(50.0)\end{array}$ & 0.010 \\
\hline $\begin{array}{l}\text { Which doctor you think spends more time with their } \\
\text { patients? }\end{array}$ & $\begin{array}{l}n \\
(\%)\end{array}$ & $\begin{array}{c}73 \\
(42.2)\end{array}$ & $\begin{array}{c}38 \\
(22.0)\end{array}$ & $\begin{array}{c}62 \\
(35.8)\end{array}$ & $\begin{array}{c}27 \\
(50.0)\end{array}$ & $\begin{array}{c}14 \\
(25.9)\end{array}$ & $\begin{array}{c}13 \\
(24.1)\end{array}$ & 0.276 \\
\hline You are embarrassed to see a gynecologist who is? & $\begin{array}{l}n \\
(\%)\end{array}$ & $\begin{array}{c}45 \\
(26.0)\end{array}$ & $\begin{array}{c}116 \\
(67.1)\end{array}$ & $\begin{array}{c}12 \\
(6.9)\end{array}$ & $\begin{array}{c}21 \\
(38.9)\end{array}$ & $\begin{array}{c}33 \\
(61.1)\end{array}$ & $\begin{array}{c}0 \\
(0.0)\end{array}$ & 0.043 \\
\hline You are embarrassed to see an obstetrician who is? & $\begin{array}{l}\mathrm{n} \\
(\%)\end{array}$ & $\begin{array}{c}43 \\
(24.9)\end{array}$ & $\begin{array}{c}112 \\
(64.7)\end{array}$ & $\begin{array}{c}18 \\
(10.4)\end{array}$ & $\begin{array}{c}19 \\
(35.2)\end{array}$ & $\begin{array}{c}34 \\
(63.0)\end{array}$ & $\begin{array}{c}1 \\
(1.9)\end{array}$ & 0.073 \\
\hline $\begin{array}{l}\text { Which one understands women's health issues } \\
\text { better? }\end{array}$ & $\begin{array}{l}n \\
(\%)\end{array}$ & $\begin{array}{c}43 \\
(24.9)\end{array}$ & $\begin{array}{c}33 \\
(19.1)\end{array}$ & $\begin{array}{c}97 \\
(56.1)\end{array}$ & $\begin{array}{c}12 \\
(22.2)\end{array}$ & $\begin{array}{c}11 \\
(20.4)\end{array}$ & $\begin{array}{c}31 \\
(57.4)\end{array}$ & 0.920 \\
\hline $\begin{array}{l}\text { Which tends to be more knowledgeable about } \\
\text { women's health? }\end{array}$ & $\begin{array}{l}n \\
(\%)\end{array}$ & $\begin{array}{c}58 \\
(33.5)\end{array}$ & $\begin{array}{c}31 \\
(17.9)\end{array}$ & $\begin{array}{c}84 \\
(48.6)\end{array}$ & $\begin{array}{c}19 \\
(35.2)\end{array}$ & $\begin{array}{c}14 \\
(25.9)\end{array}$ & $\begin{array}{c}21 \\
(38.9)\end{array}$ & 0.334 \\
\hline Which one provides better health care? & $\begin{array}{l}n \\
(\%)\end{array}$ & $\begin{array}{c}72 \\
(4 I .6)\end{array}$ & $\begin{array}{c}44 \\
(25.4)\end{array}$ & $\begin{array}{c}57 \\
(32.9)\end{array}$ & $\begin{array}{c}30 \\
(55.6)\end{array}$ & $\begin{array}{c}12 \\
(22.2)\end{array}$ & $\begin{array}{c}12 \\
(22.2)\end{array}$ & 0.173 \\
\hline Which one is more organized? & $\begin{array}{l}\mathrm{n} \\
(\%)\end{array}$ & $\begin{array}{c}70 \\
(40.5)\end{array}$ & $\begin{array}{c}61 \\
(35.3)\end{array}$ & $\begin{array}{c}42 \\
(24.3)\end{array}$ & $\begin{array}{c}23 \\
(42.6)\end{array}$ & $\begin{array}{c}18 \\
(33.3)\end{array}$ & $\begin{array}{c}13 \\
(24.1)\end{array}$ & 0.956 \\
\hline Which one do you trust more? & $\begin{array}{l}\mathrm{n} \\
(\%)\end{array}$ & $\begin{array}{c}72 \\
(4 I .6)\end{array}$ & $\begin{array}{c}41 \\
(23.7)\end{array}$ & $\begin{array}{c}60 \\
(34.7)\end{array}$ & $\begin{array}{c}24 \\
(44.4)\end{array}$ & $\begin{array}{c}17 \\
(31.5)\end{array}$ & $\begin{array}{c}13 \\
(24.1)\end{array}$ & 0.287 \\
\hline $\begin{array}{l}\text { If you are seeing a Saudi OB-GYN, who would you } \\
\text { prefer? }\end{array}$ & $\begin{array}{l}\mathrm{n} \\
(\%)\end{array}$ & $\begin{array}{c}56 \\
(32.4)\end{array}$ & $\begin{array}{c}23 \\
(13.3)\end{array}$ & $\begin{array}{c}94 \\
(54.3)\end{array}$ & $\begin{array}{c}15 \\
(27.8)\end{array}$ & $\begin{array}{c}12 \\
(22.2)\end{array}$ & $\begin{array}{c}27 \\
(50.0)\end{array}$ & 0.279 \\
\hline $\begin{array}{l}\text { If you are seeing a Non-Saudi OB-GYN who would } \\
\text { you prefer? }\end{array}$ & $\begin{array}{l}n \\
(\%)\end{array}$ & $\begin{array}{c}59 \\
(34.1)\end{array}$ & $\begin{array}{c}21 \\
(12.1)\end{array}$ & $\begin{array}{c}93 \\
(53.8)\end{array}$ & $\begin{array}{c}11 \\
(20.4)\end{array}$ & $\begin{array}{c}14 \\
(25.9)\end{array}$ & $\begin{array}{c}29 \\
(53.7)\end{array}$ & 0.022 \\
\hline
\end{tabular}

related to "embarrassment" and "knowledge about women's health." No other factors (marital status, nationality, parity, education, income) were related to women's preferences. Nearly three-quarters $(71 \%)$ of respondents age 35 or younger reported they were embarrassed to see an OB-GYN who was male $(\mathrm{p}=0.01)$ and nearly $80 \%$ of this subgroup felt that female OB-GYN physicians were more knowledgeable about women's health issues $(\mathrm{p}=0.04)$.

Figure 1 describes the classification tree analysis to test the significance of various demographic characteristics. Only age found significantly related to "embarrassment" and "knowledge about women health" and all other factors (marital status, nationality, parity, education and Income have not shown any significance. Node 1: About $71.2 \%$ or respondents with age $\leq 35$ reported that they are embarrassed to see and obstetrician who is male (P-value $=0.01)$ and $79.7 \%$ of this subgroup feels that female doctors are more knowledgeable about women's health (P-value=0.041).

\section{Discussion}

Aside from the clear overall preference in this population for a female OB-GYN physician, the most important findings were that younger and lower-income Saudi women were 
Table 4 Comparisons by Age

\begin{tabular}{|c|c|c|c|c|c|c|c|c|}
\hline & & \multicolumn{6}{|c|}{ Age in Years } & \multirow[b]{3}{*}{ P-value } \\
\hline & & \multicolumn{3}{|c|}{35 and Less } & \multicolumn{3}{|c|}{36 and Above } & \\
\hline & & $\begin{array}{c}\text { No } \\
\text { Preference }\end{array}$ & Male & Female & $\begin{array}{c}\text { No } \\
\text { Preference }\end{array}$ & Male & Female & \\
\hline Pelvic Exam & $\begin{array}{l}n \\
(\%)\end{array}$ & $\begin{array}{c}39 \\
(26.7)\end{array}$ & $\begin{array}{c}7 \\
(4.8)\end{array}$ & $\begin{array}{c}100 \\
(68.5)\end{array}$ & $\begin{array}{c}21 \\
(25.9)\end{array}$ & $\begin{array}{c}13 \\
(16.0)\end{array}$ & $\begin{array}{c}47 \\
(58.0)\end{array}$ & 0.015 \\
\hline OB Care & $\begin{array}{l}\mathrm{n} \\
(\%)\end{array}$ & $\begin{array}{c}36 \\
(24.7)\end{array}$ & $\begin{array}{c}14 \\
(9.6)\end{array}$ & $\begin{array}{c}96 \\
(65.8)\end{array}$ & $\begin{array}{c}24 \\
(29.6)\end{array}$ & $\begin{array}{c}14 \\
(17.3)\end{array}$ & $\begin{array}{c}43 \\
(53.1)\end{array}$ & 0.113 \\
\hline Gyn. Surgery & $\begin{array}{l}\mathrm{n} \\
(\%)\end{array}$ & $\begin{array}{c}45 \\
(30.8)\end{array}$ & $\begin{array}{c}16 \\
(11.0)\end{array}$ & $\begin{array}{c}85 \\
(58.2)\end{array}$ & $\begin{array}{c}28 \\
(34.6)\end{array}$ & $\begin{array}{c}19 \\
(23.5)\end{array}$ & $\begin{array}{c}34 \\
(42.0)\end{array}$ & 0.017 \\
\hline $\begin{array}{l}\text { Which doctor you think spends more time with their } \\
\text { patients? }\end{array}$ & $\begin{array}{l}n \\
(\%)\end{array}$ & $\begin{array}{c}59 \\
(40.4)\end{array}$ & $\begin{array}{c}35 \\
(24.0)\end{array}$ & $\begin{array}{c}52 \\
(35.6)\end{array}$ & $\begin{array}{c}41 \\
(50.6)\end{array}$ & $\begin{array}{c}17 \\
(21.0)\end{array}$ & $\begin{array}{c}23 \\
(28.4)\end{array}$ & 0.324 \\
\hline You are embarrassed to see a gynecologist who is? & $\begin{array}{l}n \\
(\%)\end{array}$ & $\begin{array}{c}37 \\
(25.3)\end{array}$ & $\begin{array}{c}105 \\
(71.9)\end{array}$ & $\begin{array}{c}4 \\
(2.7)\end{array}$ & $\begin{array}{c}29 \\
(35.8)\end{array}$ & $\begin{array}{c}44 \\
(54.3)\end{array}$ & $\begin{array}{c}8 \\
(9.9)\end{array}$ & 0.009 \\
\hline You are embarrassed to see an obstetrician who is? & $\begin{array}{l}n \\
(\%)\end{array}$ & $\begin{array}{c}35 \\
(24.0)\end{array}$ & $\begin{array}{c}104 \\
(71.2)\end{array}$ & $\begin{array}{c}7 \\
(4.8)\end{array}$ & $\begin{array}{c}27 \\
(33.3)\end{array}$ & $\begin{array}{c}42 \\
(51.9)\end{array}$ & $\begin{array}{c}12 \\
(14.8)\end{array}$ & 0.004 \\
\hline $\begin{array}{l}\text { Which one understands women's health issues } \\
\text { better? }\end{array}$ & $\begin{array}{l}n \\
(\%)\end{array}$ & $\begin{array}{c}37 \\
(25.3)\end{array}$ & $\begin{array}{c}23 \\
(15.8)\end{array}$ & $\begin{array}{c}86 \\
(58.9)\end{array}$ & $\begin{array}{c}18 \\
(22.2)\end{array}$ & $\begin{array}{c}21 \\
(25.9)\end{array}$ & $\begin{array}{c}42 \\
(51.9)\end{array}$ & 0.178 \\
\hline $\begin{array}{l}\text { Which tends to be more knowledgeable about } \\
\text { women's health? }\end{array}$ & $\begin{array}{l}\mathrm{n} \\
(\%)\end{array}$ & $\begin{array}{c}47 \\
(32.2)\end{array}$ & $\begin{array}{c}26 \\
(I 7.8)\end{array}$ & $\begin{array}{c}73 \\
(50.0)\end{array}$ & $\begin{array}{c}30 \\
(37.0)\end{array}$ & $\begin{array}{c}19 \\
(23.5)\end{array}$ & $\begin{array}{c}32 \\
(39.5)\end{array}$ & 0.295 \\
\hline Which one provides better health care? & $\begin{array}{l}\mathrm{n} \\
(\%)\end{array}$ & $\begin{array}{c}67 \\
(45.9)\end{array}$ & $\begin{array}{c}32 \\
(21.9)\end{array}$ & $\begin{array}{c}47 \\
(32.2)\end{array}$ & $\begin{array}{c}35 \\
(43.2)\end{array}$ & $\begin{array}{c}24 \\
(29.6)\end{array}$ & $\begin{array}{c}22 \\
(27.2)\end{array}$ & 0.412 \\
\hline Which one is more organized? & $\begin{array}{l}n \\
(\%)\end{array}$ & $\begin{array}{c}61 \\
(41.8)\end{array}$ & $\begin{array}{c}49 \\
(33.6)\end{array}$ & $\begin{array}{c}36 \\
(24.7)\end{array}$ & $\begin{array}{c}32 \\
(39.5)\end{array}$ & $\begin{array}{c}30 \\
(37.0)\end{array}$ & $\begin{array}{c}19 \\
(23.5)\end{array}$ & 0.870 \\
\hline Which one do you trust more? & $\begin{array}{l}\mathrm{n} \\
(\%)\end{array}$ & $\begin{array}{c}64 \\
(43.8)\end{array}$ & $\begin{array}{c}30 \\
(20.5)\end{array}$ & $\begin{array}{c}52 \\
(35.6)\end{array}$ & $\begin{array}{c}32 \\
(39.5)\end{array}$ & $\begin{array}{c}28 \\
(34.6)\end{array}$ & $\begin{array}{c}21 \\
(25.9)\end{array}$ & 0.056 \\
\hline $\begin{array}{l}\text { If you are seeing a Saudi OB-GYN, who would you } \\
\text { prefer? }\end{array}$ & $\begin{array}{l}\mathrm{n} \\
(\%)\end{array}$ & $\begin{array}{c}50 \\
(34.2)\end{array}$ & $\begin{array}{c}19 \\
(13.0)\end{array}$ & $\begin{array}{c}77 \\
(52.7)\end{array}$ & $\begin{array}{c}21 \\
(25.9)\end{array}$ & $\begin{array}{c}16 \\
(19.8)\end{array}$ & $\begin{array}{c}44 \\
(54.3)\end{array}$ & 0.258 \\
\hline $\begin{array}{l}\text { If you are seeing a Non-Saudi OB-GYN who would } \\
\text { you prefer? }\end{array}$ & $\begin{array}{l}n \\
(\%)\end{array}$ & $\begin{array}{c}47 \\
(32.2)\end{array}$ & $\begin{array}{c}22 \\
(15.1)\end{array}$ & $\begin{array}{c}77 \\
(52.7)\end{array}$ & $\begin{array}{c}23 \\
(28.4)\end{array}$ & $\begin{array}{c}13 \\
(16.0)\end{array}$ & $\begin{array}{c}45 \\
(55.6)\end{array}$ & 0.838 \\
\hline
\end{tabular}

more likely to prefer female OB-GYNs, while higher-income Saudi women expressed preference for male OB-GYNs. Embarrassment at utilizing a male provider was a common reason for preferring a female provider, but the most important reasons for any provider choice were experience, reputation, and board certification. Preference for female providers was strongest with regard to pelvic exams and strongest for males for gynecological surgery. These results are similar to those from previous studies.

In a span of 50 years, OB-GYN practice has experienced a remarkable shift from its male-dominant history, with female OB-GYNs comprising $7 \%$ of total providers worldwide in 1970 to a projected $66 \%$ by $2022 .{ }^{8}$ The sharp increase in the availability of female OB-GYNs is accompanied by increased demand for them in many countries, though often these preferences are situation-specific. In one of the largest studies of these trends, Tobler et al conducted a meta-analysis of over 14,000 patients worldwide, showing that half $(50.2 \%)$ preferred a female OBGYN doctor, and $8.3 \%$ preferring a male provider. The authors noted that a subgroup of nearly 10,000 American patients had similar preferences of $53.2 \%$ and $8.4 \%$, respectively. Despite expected differences due to cultural dissimilarity, a subgroup of Nigerian and Middle Eastern 
Age in Years

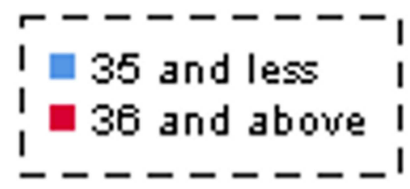

\begin{tabular}{|lrr|}
\hline \multicolumn{3}{|c|}{ Node 0} \\
Categony & $\&$ & $n$ \\
\hline 35 and less & 64.3 & 146 \\
\hline 36 and above & 35.7 & 81 \\
\hline Total & \multicolumn{3}{|c|}{100.0} & 227 \\
\hline & &
\end{tabular}

You are embarrassed to see an obstetrician who is?

Adj. P-value $=0.010$, Chi-square $=8.527$. $d f=1$

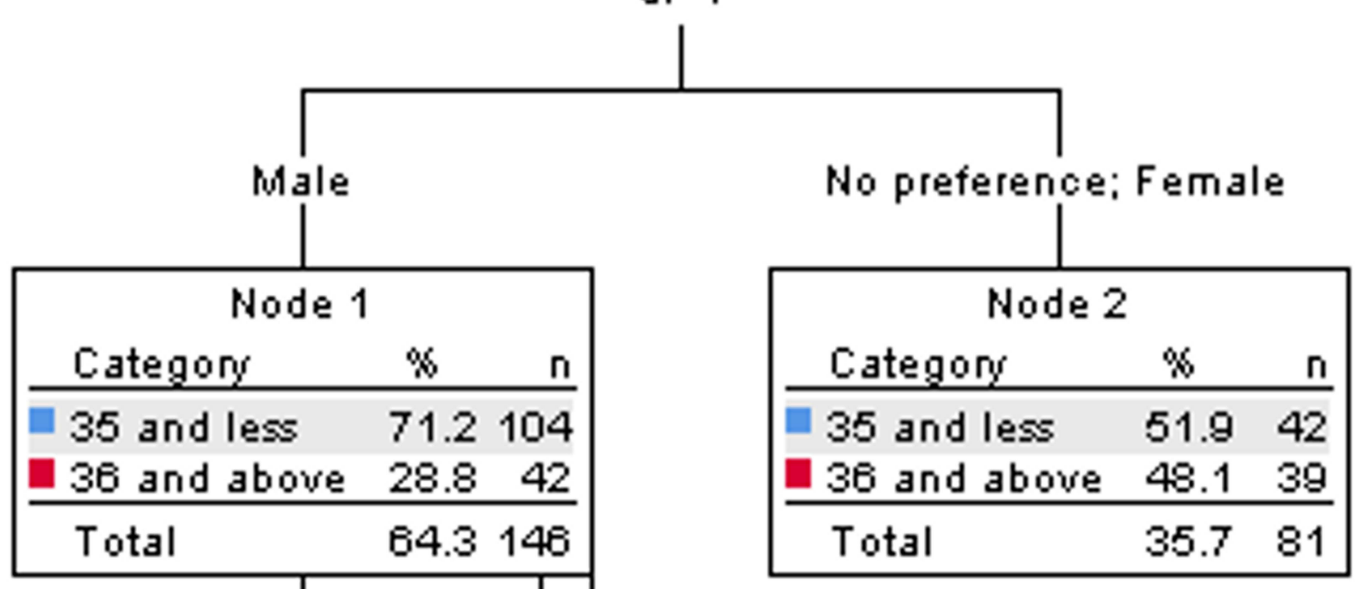

Which tends to be more knowledgeable about women's health?

Adj. P-value $=0.041$, Chi-square $=6.090$. $\mathrm{df}=1$

Male; No preference

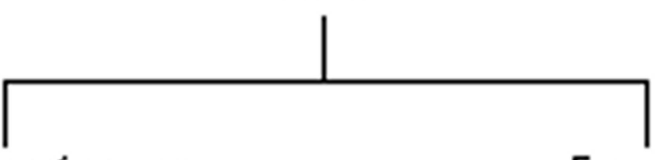

Male: No preference
\begin{tabular}{|lll|}
\hline Node 3 \\
Categony & $\&$ & $n$ \\
\hline 35 and less & 61.2 & 41 \\
36 and above & 38.8 & 26 \\
\hline Total & 29.5 & 67 \\
\hline
\end{tabular}

Female
\begin{tabular}{|ccc|}
\hline \multicolumn{3}{|c|}{ Node 4} \\
Categony & $\%$ & $n$ \\
\hline 35 and less & 79.7 & 63 \\
36 and above & 20.3 & 16 \\
\hline Total & 34.8 & 79 \\
\hline
\end{tabular}

Figure I Multivariate classification tree analysis to test the significance of various demographic characteristics about gender preferences of respondents. 
patients showed nearly the same preferences as their American counterparts. ${ }^{9}$ A study from Brazil showed that female patients were more than eight times more likely to prefer a female OB-GYN, though most had no gender preference overall. ${ }^{10}$ Data from the United Kingdom reflected a similar trend, with a 10:1 female to male provider preference, again with a majority having no gender preference. ${ }^{11}$

On the other hand, studies of OB-GYN provider gender preference for women of Islamic Middle Eastern countries have been remarkably consistent in their findings that, overall, these women prefer female providers. One study from Amir et al in 2012 found that Muslim Israeli-Arab women had no gender preference for their family physician, but over three-quarters $(77 \%)$ of respondents preferred having a female OB-GYN and $86 \%$ preferred female providers for pelvic exams. ${ }^{12}$ The reasons for their preferences were similar to those of the women in the present study, most commonly citing embarrassment with a male provider and more comfort with a female OBGYN. Additionally, despite the clear gender preference reported in the present study, experience, knowledge, and ability were far more important factors. ${ }^{12}$

Similar results to the present study were reported in a cross-sectional survey of 500 women in the neighboring nation of Iraq, where $73 \%$ of respondents preferred a female gynecologist and 79\% preferred a female obstetrician, with those preferences also declining with increasing education level. ${ }^{7}$ Correlation of female physician preference with lower education was noted in a study of 218 women from the United Arab Emirates (UAE), another neighboring nation with similar sociodemographic and cultural values as Saudi Arabia. ${ }^{13}$ With regard to OBGYN care in the UAE, 508 respondents showed preference for female OB-GYN care, primarily due to privacy concerns during the examination, a preference that decreased with increasing respondent age as in the present study. ${ }^{14}$ Studies from Lebanon and Nigeria, both of countries where Muslims make up 50\% or more of the population, indicated a strong preference for female OB-GYN clinicians among Muslim patients in particular. ${ }^{15-17}$

Compared to the results of this study, previous data about OB-GYN provider gender preference in Saudi patients have reported similar findings. In a 2015 study at a university hospital in the Saudi capital of Riyadh, 85\% of female respondents preferred a same-gender provider, and as with the current study, embarrassment was cited as the primary reason. ${ }^{18}$ A 2019 analysis of 1650 Saudi women interviewed in the community found that $86 \%$ preferred a same-gender provider for a genital exam. ${ }^{19}$ A previous study of 418 primary care patients performed at the same hospital as the current study reported that over $77 \%$ of female patients preferred a female physician for a pelvic examination, with the most common determinant being greater comfort with a female physician. ${ }^{20}$ The sheer volume and scope of routinely required intimate examinations in OB-GYN are similar to urology, a branch of medicine that also shows a marked preference for patientprovider gender concordance for similarly stated reasons as found here. ${ }^{21}$

One consistent finding in most relevant studies is an increased male preference for gynecological surgical procedures. This trend is particularly notable in older women and is perhaps predictable since older women are more likely to require gynecological surgical procedures and were more likely to have had prior exposure to a male provider. $^{22,23}$ OB-GYN is a clinical practice that includes elements of primary care, but is fundamentally a surgical specialty, with patient expectations of provider competence, confidence, and assertiveness. There is evidence that patients tend to believe that male providers have more of these traits than women do, and women who display these traits are often viewed less favorably by patients and colleagues. ${ }^{24}$ In a Stanford University study, men were $47 \%$ more likely to receive high OB-GYN patient satisfaction scores. These perceptions occur despite the presence of an overall gender preference for female OB-GYN care, which suggests that such biases are largely unconscious.

Younger women, on the other hand, have come of age in a world where the presence of female OB-GYN physicians is not only normalized but increasingly expected. For women in Saudi Arabia, this increased level of choice has critical implications for personal and public health. The expectations of communal, non-confrontational, empathetic behavior in female physicians that penalizes them for assertiveness are also highly desired characteristics in patient interactions. One meta-analysis showed that female physicians engage in a more patient-centered, empathetic style centered on partnership and cooperation. ${ }^{25}$ Furthermore, patients who are more comfortable with their providers are more likely to be forthright about their symptoms, which has a significant bearing on their health. Rates of STIs in Saudi Arabia are quite low compared to Western countries, but have risen recently. Rates of nongonococcal urethritis, for example, were reported to 
be 7.5 per 100,000 in a 2006 study, but 25.4 per 100,000 in a 2016 study. ${ }^{26,27}$ Stigma about these conditions may prevent patients from talking to their doctors about symptoms and potentially contribute to increased spread.

In the KSA, the conservative nature of Saudi culture and the centrality of Islamic principles to public life both substantially influence routine health behaviors such as the choice of physician. The primacy of the doctor-patient relationship, which is one of close trust, requires that women feel secure about such choices in OB-GYN care, and the increasing number of female OB-GYN physicians in recent years within Saudi Arabia allows Saudi women to have more choices in their care. This will likely remain the case. Though interest in the OB-GYN from Saudi medical students domestically and internationally is low, Saudi students who choose OB-GYN specialization are more likely to be women. ${ }^{28,29}$ In addition, one study of Saudi Arabian women seeking OB-GYN care showed that they were more comfortable in the presence of female medical students, especially when pelvic examination was indicated, and the presence of male medical students increased the levels of patient discomfort. ${ }^{30}$

The study's strengths include a high response rate and a high degree of internal and external data consistency. Limitations are primarily due to those inherent in crosssectional surveys and use of a single academic care center site, which may constrain the generalizability of study findings. Furthermore, respondents were not asked about all factors they utilize in choosing providers, which was limited by the available survey choices. However, the level of consistency with other studies of patients' provider preferences, especially in culturally similar countries, suggests fundamental soundness of the methodology and results.

\section{Conclusions}

This study found that women presenting to King Abdulaziz University Hospital in Jeddah, Saudi Arabia, for OB-GYN care show a strong preference for female providers, though some women prefer male providers in certain circumstances (gynecologic surgery). Despite these gender preferences, more important to women is the experience, qualification, and reputation of the OB-GYN provider. Such trends are consistent with culturally similar countries and in line with trends worldwide, and have potential to significantly impact the personal health sought by women in Saudi Arabia and perhaps elsewhere in the Middle East as well.

\section{Disclosure}

The authors report no conflicts of interest in this work.

\section{References}

1. Stewart MA. Effective physician-patient communication and health outcomes: a review. CMAJ. 1995;152(9):1423.

2. Zuckerman M, Navizedeh N, Feldman J, McCalla S, Minkoff H. Determinants of women's choice of obstetrician/gynecologist. $J$ Women's Health Genderbased Med. 2002;11(2):175-180.

3. Tsui-Fang L, Chen J. Effect of physician gender on demand for pap tests. Econ Res Int. 2014;2014. doi:10.1155/2014/647169

4. Chang JC, Odrobina MR, McIntyre-Seltman K. The effect of student gender on the obstetrics and gynecology clerkship experience. $J$ Women's Health. 2010;19(1):87-92.

5. Anfinan N, Alghunaim N, Boker A, et al. Obstetric and gynecologic patients' attitudes and perceptions toward medical students in Saudi Arabia. Oman Med J. 2014;29(2):106.

6. Buck KS, Littleton HL. Stereotyped beliefs about male and female OB-GYNS: relationship to provider choice and patient satisfaction. J Psychosomatic Obstetrics Gynecol. 2014;35(1):1-7.

7. Lafta RK. Practitioner gender preference among gynecologic patients in Iraq. Health Care Women Int. 2006;27(2):125-130.

8. Rayburn WF, editor. The Obstetrician-Gynecologist Workforce in the United States: Facts, Figures, and Implications, 2017. Washington, DC: American Congress of Obstetricians and Gynecologists; 2017.

9. Tobler KJ, Wu J, Khafagy AM, Pier BD, Torrealday S, Londra L. Gender preference of the obstetrician gynecologist provider: a systematic review and meta-analysis [1E]. Obstet Gynecol. 2016;127:43S.

10. Wanderley M, Sobral DT. Ob-Gyn gender preferences of gynecology ambulatory patients and students' choice of the specialty. Revista Brasileira de Ginecologia e Obstetricia. 2017;39(12):645-646.

11. Makam A, Saroja CSM, Edwards G. Do women seeking care from obstetrician-gynaecologists prefer to see a female or a male doctor? Arch Gynecol Obstet. 2010;281(3):443-447.

12. Amir H, Tibi Y, Groutz A, Amit A, Azem F. Unpredicted gender preference of obstetricians and gynecologists by Muslim Israeli-Arab women. Patient Educ Couns. 2012;86(2):259-263.

13. McLean M, Al Yahyaei F, Al Mansoori M, Al Ameri M, Al Ahbabi S, Bernsen R. Muslim women's physician preference: beyond obstetrics and gynecology. Health Care Women Int. 2012;33 (9):849-876.

14. Rizk DE, El-Zubeir MA, Al-Dhaheri AM, Al-Mansouri FR, AlJenaibi HS. Determinants of women's choice of their obstetrician and gynecologist provider in the UAE. Acta Obstet Gynecol Scand. 2005;84(1):48-53.

15. Kettani H. Muslim population in Africa: 1950-2020. Int J Environ Sci Devel. 2010;1(2):136.

16. Mattar S, Nassar A, Ghulmiyyah L, Haddad S, Tamim H, Hobeika E. Factors that affect women's choice of their obstetrician and gynecologist: a survey of Lebanese women. Clin Exp Obstet Gynecol. 2019;46(3):408-412.

17. Bukar M. Women's gender preferences for their obstetrician and gynaecologist at the University of Maiduguri Teaching Hospital, Maiduguri, Nigeria. Medicine. 2012;5:6.

18. Mandil AM, Alhayyan RM, Alshalawi AA, Alemran AS, Alayed MM. Preference of physicians' gender among male and female primary health care clinic attendees in a university hospital in Saudi Arabia. Saudi Med J. 2015;36(8):1011.

19. Alyahya G, Almohanna H, Alyahya A, et al. Does physicians' gender have any influence on patients' choice of their treating physicians? J Nat Sci Med. 2019;2(1):29. 
20. Shamrani H. A cross-sectional survey of women's provider gender preferences for gynecology and obstetrics care at King Abdulaziz University Hospital. J Womens Health Care. 2016;5:347-354.

21. Amir H, Beri A, Yechiely R, Amir Levy Y, Shimonov M, Groutz A. Do urology male patients prefer same-gender urologist? Am J Men's Health. 2018;12(5):1379-1383.

22. Rock JA. Quality-of-life assessment in gynecologic surgery. J Reprod Med. 2001;46(5 Suppl):515-519.

23. Briët JM, Mourits MJ, van Leeuwen BL, et al. Age should not be a limiting factor in laparoscopic surgery: a prospective multicenter cohort study on quality of life after laparoscopic hysterectomy. Clin Interv Aging. 2018;13:2517.

24. Carnes M, Bartels CM, Kaatz A, Kolehmainen C. Why is John more likely to become department chair than Jennifer? Trans Am Clin Climatol Assoc. 2015;126:197.

25. Jefferson L, Bloor K, Birks Y, Hewitt C, Bland M. Effect of physicians' gender on communication and consultation length: a systematic review and meta-analysis. J Health Serv Res Policy. 2013;18(4):242-248.
26. Madani TA. Sexually transmitted infections in Saudi Arabia. BMC Infect Dis. 2006;6(1):3.

27. Memish ZA, Filemban SM, Al-Hakeem RF, Hassan MH, Al-Tawfiq JA. Sexually transmitted infections case notification rates in the Kingdom of Saudi Arabia, 2005-2012. The Journal of Infection in Developing Countries. 2016;10(08):884-887. doi:10.3855/jidc.7020

28. Abu-Rafea BF, Al-Hassan BF, Al Nakshabandi KA, Rahbini NO, Al-Shaikh GK. Factors influencing students' decision in choosing obstetrics and gynecology as a career in a university hospital in Central Saudi Arabia. Saudi Med J. 2011;32(7):730-734.

29. Mahha AJ, Abutaleb AA, Madkhali AM, Hakami MM, Jelan NS, Hakami RH. Saudi medical students' views on obstetrics and gynecology as a future career specialty. J Fam Med PrimCare. 2020;9 (2):859.

30. Subki AH, Algethami MR, Addas FA, Alnefaie MN, Hindi MM, Abduljabbar HS. Women's perception and attitude to medical students' participation in obstetrics and gynecology care. Saudi Med J. 2018;39(9):902-909.
International Journal of Women's Health

\section{Publish your work in this journal}

The International Journal of Women's Health is an international, peerreviewed open-access journal publishing original research, reports, editorials, reviews and commentaries on all aspects of women's healthcare including gynecology, obstetrics, and breast cancer. The

\section{Dovepress}

manuscript management system is completely online and includes a very quick and fair peer-review system, which is all easy to use. Visit http://www.dovepress.com/testimonials.php to read real quotes from published authors. 